\title{
EXPANSIONS FOR REPEATED INTEGRALS OF PRODUCTS WITH APPLICATIONS TO THE MULTIVARIATE NORMAL
}

\author{
Christopher S. Withers ${ }^{1}$ And Saralees Nadarajah ${ }^{2}$
}

\begin{abstract}
We extend Leibniz' rule for repeated derivatives of a product to multivariate integrals of a product. As an application we obtain expansions for $P(a<Y<b)$ for $Y \sim N_{p}(0, V)$ and for repeated integrals of the density of $Y$. When $V^{-1} y>0$ in $R^{3}$ the expansion for $P(Y<y)$ reduces to one given by [H. Ruben J. Res. Nat. Bureau Stand. B 68 (1964) 3-11]. in terms of the moments of $N_{p}\left(0, V^{-1}\right)$. This is shown to be a special case of an expansion in terms of the multivariate Hermite polynomials. These are given explicitly.
\end{abstract}

Mathematics Subject Classification. 60E05, 62H05.

Received July 19, 2009. Revised 1st Febuary, 2010.

\section{INTRODUCTION AND SUMMARY}

Expansions of the multivariate normal density are commonly used in statistical theory and its applications. One most common use is to compute multinormal probabilities, for example, rectangular and orthant multinormal probabilities arising in the multivariate probit model, the multivariate ordinal response model, multivariate paired comparisons and Thurstonian models for rankings; such probabilities also arise in almost every area of science, engineering and medicine. Many of the known procedures for computing multinormal probabilities are based on series truncations. Another common use is to find the approximate distribution of a specified statistic. The exact distribution of a statistic is often too complicated even when the data are multinormal. Examples include distributions of the eigenvalues and eigenvectors of the sample covariance matrix for a normal population.

The calculation of multinormal probabilities is one reason why we need expansions for repeated integrals of products of univariate and multivariate normal densities. The need for such expansions arises in many other areas too. We mention five applications:

- in the calculation of moments of truncated normal distribution [2];

- in the expression of the non-central $t$ density [2];

- in the posterior distribution of a Poisson variate with chi-squared prior for the squared mean parameter of the Poisson variate [2];

- in the calculation of shape distributions [3,8];

Keywords and phrases. Asymptotic expansion, Leibniz' rule, repeated integrals of products, multivariate Hermite polynomials, multivariate normal.

${ }^{1}$ Applied Mathematics Group Industrial Research Limited Lower Hutt, New Zealand

${ }^{2}$ School of Mathematics University of Manchester Manchester M13 9PL, UK. mbbsssn2@manchester.ac.uk 
- in directional statistics with respect to computing the mean resultant length of the spherically projected normal distribution [10].

Each of these applications involves explicitly repeated integrals of products of univariate and multivariate normal densities. We refer the readers to the cited references for further details.

There is a large literature on expansions for the multivariate normal distribution. We refer the readers to Tong [14], von Rosen ([15], Sects. 3 and 4), Kotz et al. ([6], Chap. 45), Kotz and Nadarajah ([7], Chap. 6), Kollo and von Rosen ([5], Chap. 1, p. 152) and Kollo and von Rosen ([5], Chap. 3) for comprehensive reviews. Further motivation for expansions of the multivariate normal can be found in these references.

The aim of this paper is to provide expansions based on an extension of Leibniz' rule. The results are organised as follows. In Section 2 we extend Leibniz' rule for differentiating a product to obtain an expansion for repeated integrals of a product of $q$ functions $\phi_{1}(y) \ldots \phi_{q}(y)$ from $C^{p}$ to $C$, the complex numbers, in terms of the repeated integrals of $\phi_{1}$ and the derivatives of $\phi_{2}, \ldots, \phi_{q}$. For example, if $q=2$ and $\phi_{1}(y)=1$ and $\phi_{2}$ is the density of a random variable $Y$ in $R^{p}$ for $R$, the reals, this gives $2^{p}$ Taylor series expansions for $P(x<Y<y)$ in powers of $y-x$. These expansions are given in Section 3 together with expansions about zero. In Section 4 we take $\phi_{1}(y)=\exp \left(y^{\prime} z\right)$ and $\phi_{2}=\phi_{V}$, the density of $Y \sim N_{p}(0, V)$. We obtain expansions in inverse powers of $z=V^{-1} \mu$ for the $2^{p}$ probabilities $P\left(Y_{j}>\right.$ or $\left.<\mu_{j}, 1 \leq j \leq p\right)$ in terms of the multivariate Hermite polynomials and the moments of $N_{p}\left(0, V^{-1}\right)$. These polynomials and moments are given in the appendix. The special case $P(Y<\mu)$ for $z>0$ was obtained by [11].

We use the following notation: $N=\{0,1,2, \ldots\}, Z=\{\ldots,-1,0,1, \ldots\}, \bar{C}$ is the closure of $C$, that is, it includes all points at $\infty$. For $y$ in $C$ and $\lambda$ in $N$,

$$
(y)_{\lambda}=y(y-1) \cdots(y-\lambda+1)=\Gamma(y+1) / \Gamma(y-\lambda+1)
$$

and $\left(\begin{array}{l}y \\ \lambda\end{array}\right)=(y)_{\lambda} / \lambda$ ! the binomial coefficient, so

$$
\left(\begin{array}{c}
-y \\
\lambda
\end{array}\right)=(-1)^{\lambda}\left(\begin{array}{c}
y-1+\lambda \\
\lambda
\end{array}\right)
$$

Fix $y_{0}$ in $\bar{C}^{p}$. For $y, z$ in $\bar{C}^{p}, \lambda$ in $N^{p}$ and $v$ in $Z^{p}$ set

$$
\begin{aligned}
& y^{z}=\prod_{j=1}^{p} y_{j}^{z_{j}},(y)_{\lambda}=\prod_{j=1}^{p}\left(y_{j}\right)_{\lambda_{j}},\left(\begin{array}{c}
y \\
\lambda
\end{array}\right)=\prod_{j=1}^{p}\left(\begin{array}{c}
y_{j} \\
\lambda_{j}
\end{array}\right), \\
& \lambda !=\prod_{j=1}^{p} \lambda_{j} !, \Gamma(y)=\prod_{j=1}^{p} \Gamma\left(y_{j}\right), \partial_{y}^{v}=\prod_{j=1}^{p} \partial_{y_{j}}^{v_{j}}
\end{aligned}
$$

where $\partial_{y}=\partial / \partial y, \partial_{y}^{-1}=\int_{y_{0}}^{y} \mathrm{~d} y=(-1)^{p} \int_{y}^{y_{0}} \mathrm{~d} y$, so $\partial_{y_{j}}^{-1}=\int_{y_{0}}^{y_{j}} \mathrm{~d} y_{j}, y \times z=\left(y_{1} z_{1}, \ldots, y_{p} z_{p}\right)^{\prime}, \operatorname{Re} z=$ $\left(\operatorname{Re} z_{1}, \ldots, \operatorname{Re} z_{p}\right)^{\prime},|v|=\sum_{1}^{p} v_{j}$ and $(-)^{V}=(-1)^{|V|}$. In $N^{p}, 1$ is the vector of 1 's and 0 is the vector of 0 's. For $\phi: C^{p} \rightarrow C$ a given function,

$$
H_{\lambda}(y)=\phi(y)^{-1}\left(-\partial_{y}\right)^{\lambda} \phi(y)
$$

For $y, z$ in $R^{p}, \operatorname{sign} y=\left(\operatorname{sign} y_{1}, \ldots, \operatorname{sign} y_{p}\right)^{\prime}, m(y, z)=\left(m\left(y_{1}, z_{1}\right), \ldots, m\left(y_{p}, z_{p}\right)\right)^{\prime}$ for $m=\min$ or $\max , y<z$ means $y_{j}<z_{j}$ for $1 \leq j \leq p, y \leq z$ means $y_{j} \leq z_{j}$ for $1 \leq j \leq p, \sum_{\lambda}$ sums over $\lambda$ in $N^{p}$. We shall also denote $\phi_{j}^{(\lambda)}(y)=\partial_{y}^{\lambda} \phi_{j}(y)$ for $j=1,2, \ldots, q$. 


\section{LEIBNIZ' RULE FOR INTEGRALS}

Theorem 2.1 extends Leibniz' rule for the product of $q$ functions.

Theorem 2.1. Leibniz' rule for differentiating a product can be written

$$
\partial_{y}^{v} \phi_{1}(y) \phi_{2}(y)=\sum_{\lambda}\left(\begin{array}{l}
v \\
\lambda
\end{array}\right) \phi_{1}^{(v-\lambda)}(y) \phi_{2}^{(\lambda)}(y)
$$

for $v$ in $N^{p}$, where $\left\{\phi_{n}\right\}$ are functions from $C^{p}$ to $C$ and $y \in C^{p}$. Repeated application of (2.1) implies for $v$ in $N^{p}$

$$
\partial_{y}^{v} \prod_{n=1}^{q} \phi_{n}(y)=\sum_{\lambda}^{v} v ! \prod_{n=1}^{q} \phi_{n}^{\left(\lambda_{n}\right)}(y) / \lambda_{n} !
$$

or equivalently

$$
\partial_{y}^{v} \prod_{n=1}^{q} \phi_{n}(y)=\sum_{\lambda_{2}} \cdots \sum_{\lambda_{q}}(v)_{\lambda_{1}} \phi_{1}^{\left(\lambda_{1}\right)}(y) \prod_{n=2}^{q} \phi_{n}^{\left(\lambda_{n}\right)}(y) / \lambda_{n} !
$$

where $\sum_{\lambda}^{v}$ sums over $\lambda_{1}, \ldots, \lambda_{q} \in N^{p}$ such that $v=\sum_{n=1}^{q} \lambda_{n}$. Both (2.1) and (2.3) hold as asymptotic expansions for $v$ in $Z^{p}$ provided that for $\left\{\lambda_{n}\right\}$ of $(2.3)$ with $\left(\begin{array}{l}v \\ \lambda\end{array}\right) \neq 0$ and $1 \leq j \leq p$,

$$
\text { if } v_{j}<0 \text { then } \prod_{n=1}^{q} \phi_{n}^{\left(\lambda_{n}\right)}(y) \rightarrow 0
$$

as $y_{j} \rightarrow y_{0 j}$.

Proof. Suppose first $p=1$. Integrating by parts if $\phi_{2}(y) \partial_{y}^{-1} \phi_{1}(y) \rightarrow 0$ as $y \rightarrow y_{0}$ then $\partial_{y}^{-1} \phi_{1}(y) \phi_{2}(y)=$ $\phi_{2}(y) \partial_{y}^{-1} \phi_{1}(y)-\partial_{y}^{-1}\left\{\phi_{2}^{(1)}(y) \partial_{y}^{-1} \phi_{1}(y)\right\}$ which we write as $I=A+I B$ on $\phi_{1} \phi_{2}$, where $I_{y}=\partial_{y}^{-1}, A_{y}=\partial_{1 y}^{-1}$, $B_{y}=-\partial_{2 y} \partial_{1 y}^{-1}$ and $\partial_{n y}$ is $\partial_{y}$ acting on $\phi_{n}$ only. So, if $\phi_{2}^{(n-1)}(y) \partial_{y}^{-n} \phi_{1}(y) \rightarrow 0$ as $y \rightarrow y_{0}$ for $1 \leq n \leq m$ then

$$
\begin{aligned}
I & =A \sum_{n=0}^{m-1} B^{n}+I B^{m} \text { on } \phi_{1} \phi_{2} \\
& =A(1-B)^{-1} \text { if } m=\infty \text { and } I B^{m} \phi_{1} \phi_{2} \rightarrow 0 \text { as } m \rightarrow \infty
\end{aligned}
$$

So, for $v=-n<0$, since $A$ and $B$ commute, (2.4) at $q=2$ implies

$$
\partial^{v} \phi_{1} \phi_{2}=I^{n} \phi_{1} \phi_{2}=A^{n}(1-B)^{-n} \phi_{1} \phi_{2}=A^{n} \sum_{\lambda}\left(\begin{array}{c}
-n \\
\lambda
\end{array}\right)(-B)^{\lambda} \phi_{1} \phi_{2},
$$

which is equal to the right hand side of (2.1) at $y$. So, (2.1) holds for $v$ in $Z^{p}$ provided that for $1 \leq j \leq n$, $\left(I B^{m}\right)^{j} \phi_{1} \phi_{2} \rightarrow 0$ as $m \rightarrow \infty$. If we only seek asymptotic expansions and are not primarily interested in convergence, we may ignore this condition.

For $p \geq 1,(2.1)$ now follows from $\partial_{y}^{v}=\prod_{j=1}^{p} \partial_{y_{j}}^{v_{j}}$. Replacing $\phi_{2}$ by $\phi_{2} \cdots \phi_{q}$ and applying (2.2) with $v=\lambda$, we obtain (2.3) for $v$ in $Z^{p}$. 
Note 2.1. By reversal of order in integration for $m \geq 1$ in $N^{p}$,

$$
\partial_{y}^{-m} \phi_{1}(y)=\int_{y_{0}}^{y}(y-x)^{m-1} \phi_{1}(x) \mathrm{d} x /(m-1) !
$$

or equivalently $\left(-\partial_{y}\right)^{-m} \phi_{1}(y)=\int_{y}^{y_{0}}(x-y)^{m-1} \phi_{1}(x) \mathrm{d} x /(m-1)$ ! so (2.1) with $v=-n$ and (1.1) imply for $n \geq 0$ in $N^{p}$,

$$
\begin{aligned}
n !\left(\int_{y_{0}}^{y} \mathrm{~d} y\right)^{n+1} \phi_{1}(y) \phi(y) & =\int_{y_{0}}^{y}(y-x)^{n} \phi_{1}(x) \phi(x) \mathrm{d} x \\
& =\sum_{\lambda}(-)^{|\lambda|} \phi^{(\lambda)}(y)(\lambda !)^{-1} \int_{y_{0}}^{y}(y-x)^{n+\lambda} \phi_{1}(x) \mathrm{d} x \\
& =\phi(y) \sum_{\lambda} H_{\lambda}(y)(\lambda !)^{-1} \int_{y_{0}}^{y}(y-x)^{n+\lambda} \phi_{1}(x) \mathrm{d} x
\end{aligned}
$$

for $H_{\lambda}(y)$ of (1.2).

Note 2.2. It should be possible to extend (2.1) and (2.3) to $v$ in $C^{p}$ using

$$
\phi_{1}^{(v)}(y)=(2 \pi i)^{-p} \Gamma(v+1) \int(y-x)^{-v-1} \phi_{1}(x) \mathrm{d} x,
$$

where $x_{j}$ is integrated along a contour in $C$ around $y_{j}$. For $v_{j}$ in $\{-1,-2, \ldots\}$, one would use (2.5) instead.

Note 2.3. Now suppose that the derivatives of $\phi_{2}, \ldots, \phi_{n},\left\{\phi_{n}^{(\lambda)}(y), n \geq 2, \lambda\right.$ in $\left.N^{p}\right\}$, are each bounded near $y=y_{0}$. Then (2.4) holds if for $\lambda \leq v$ and $1 \leq j \leq p$

$$
\text { if }\left(\begin{array}{l}
v \\
\lambda
\end{array}\right) \neq 0 \text { and } v_{j}<0 \text { then } \phi_{1}^{(\lambda)}(y) \rightarrow 0 \text { as } y \rightarrow y_{0} .
$$

This holds if $v \leq-1$ and the right hand side of (2.5) is bounded near $y=y_{0}$ for $m \geq 1$. For $v$ in $Z^{p}$ set $J=\left\{1 \leq j \leq p: v_{j}<0\right\}$ and $K=\left\{1 \leq j \leq p: v_{j} \geq 0\right\}$. Then $n_{j}=-\lambda_{j}-1 \geq 0$ for $j$ in $J$ if $\lambda_{j} \geq v_{j}$ by $(2.5)$. So,

$$
\left\{\lambda_{j} \leq v_{j}<0, j \in J\right\} \text { and }\left\{\lambda_{j}>0, j \in K\right\}
$$

implies

$$
\phi_{1}^{(\lambda)}(y)=\prod_{j \in J} \int_{y_{0}}^{y_{j}}\left(y_{j}-x_{j}\right)^{n_{j}} \phi_{1}^{\left(\lambda_{K}\right)}(x) \mathrm{d} x_{j} / n_{j} !
$$

where $x_{k}=y_{k}$ for $k$ in $K$ and $\phi_{1}^{\left(\lambda_{K}\right)}(x)=\prod_{k \in K} \partial_{x_{k}}^{\lambda_{k}} \phi_{1}(x)$. So, (2.6) holds if (2.7) implies that the right hand side of (2.8) is bounded near $y=y_{0}$. This illustrates the purely notational difficulty of applying (2.1) for $v$ in $Z^{p}$ as opposed to the cases $v \geq 0$ or $v \leq-1$.

We now set $q=2, \phi=\phi_{2}$ and fix $z$ in $C^{p}$. 
Example 2.1. Take $\phi_{1}(y)=1$ and $y_{0}$ in $C^{p}$. By $(2.5), \partial_{y}^{-m} 1=\left(y-y_{0}\right)^{m} / m$ ! for $m \in N^{p}$, so by (2.1), for $n$ in $N^{p}$

$$
\begin{aligned}
\partial_{y}^{-n} \phi(y) & =\left(\int_{y_{0}}^{y} \mathrm{~d} y\right)^{n} \phi(y)=\sum_{\lambda}\left(\begin{array}{c}
-n \\
\lambda
\end{array}\right) \phi^{(\lambda)}(y)\left(y-y_{0}\right)^{n+\lambda} /(n+\lambda) ! \\
& =(n-1) !^{-1} \phi(y) \sum_{\lambda} H_{\lambda}(y)\left(y-y_{0}\right)^{n+\lambda}(n+\lambda)^{-1} / \lambda !
\end{aligned}
$$

if also $n \geq 1$. If we compare this with

$$
\partial_{y}^{-n} \phi(y)=\left(\int_{y_{0}}^{y} \mathrm{~d} y\right)^{n} \phi(y)=\sum_{\lambda} \phi\left(y_{0}\right)\left(y-y_{0}\right)^{\lambda+n} /(\lambda+n) !
$$

obtained by expanding $\phi(y)$ about $y_{0}$ and multiplying by $\partial_{y}^{n}$, we obtain the identity

$$
\sum_{j=0}^{\lambda}(-)^{j} /\{(n+j) !(\lambda-j) !\}=\sum_{k=0}^{\lambda}(-)^{\lambda-k} /\{k !(\lambda+n-k) !\}=1 /\{(\lambda+n) \lambda !(n-1) !\}
$$

which we have not managed to prove directly. To state the extension of (2.1) to general $v$ in $Z^{p}$, one uses

$$
\phi_{1}^{(v-\lambda)}(y)=I\left(\lambda_{k}=v_{k} \text { for } k \in K\right) \prod_{j \in J}\left(y_{j}-y_{0 j}\right)^{m_{j}} / m_{j} !
$$

where now $m=\lambda-v, J=\left\{1 \leq j \leq p: m_{j}>0\right\}$ and $K=\left\{1 \leq j \leq p: m_{j} \leq 0\right\}$.

Example 2.2. Take $\phi_{1}(y)=\exp \left(y^{\prime} z\right)$, where $z_{1}, \ldots, z_{p} \neq 0$. For $p=1, n \in Z$ and $-\infty \leq y_{0} \operatorname{Re} z<\infty$,

$$
\partial_{y}^{-n} \exp (y z)=z^{-n} \exp (y z) A_{n}\left(z\left(y-y_{0}\right)\right)
$$

where

$$
A_{n}(h)= \begin{cases}1-\exp (-h) \sum_{j=0}^{n} h^{j} / j !, & \text { for } n>0 \text { and } h \text { finite } \\ 1, & \text { for } n \leq 0 \text { or } \operatorname{Re} h=\infty\end{cases}
$$

So, for $p \geq 1, n$ in $Z^{p}$ and $-\infty \leq y_{0} \times \operatorname{Re} z<\infty$,

$$
\partial_{y}^{n} \exp \left(y^{\prime} z\right)=z^{-n} \exp \left(y^{\prime} z\right) A_{n}\left(z \times\left(y-y_{0}\right)\right),
$$

where

$$
A_{n}(h)=\prod_{j=1}^{p} A_{n_{j}}\left(h_{j}\right)
$$

So,

$$
\begin{aligned}
\left(\int_{y_{0}}^{y} \mathrm{~d} y\right)^{n} \phi(y) \exp \left(y^{\prime} z\right) & =\exp \left(y^{\prime} z\right) \sum_{\lambda}\left(\begin{array}{c}
-n \\
\lambda
\end{array}\right) \psi^{(\lambda)}(y) z^{-n-\lambda} A_{n+\lambda}\left(z \times\left(y-y_{0}\right)\right) \\
& =\phi(y) \exp \left(y^{\prime} z\right) \sum_{\lambda}\left(\begin{array}{c}
n-1+\lambda \\
\lambda
\end{array}\right) H_{\lambda}(y) z^{-n-\lambda} A_{n+\lambda}\left(z \times\left(y-y_{0}\right)\right)
\end{aligned}
$$


or equivalently, for $n$ in $Z^{p}$

$$
\partial_{y}^{n} \phi(y) \exp \left(y^{\prime} z\right)=\exp \left(y^{\prime} z\right) \sum\left(\begin{array}{l}
n \\
\lambda
\end{array}\right) \phi^{(\lambda)}(y) z^{n-\lambda} A_{-n+\lambda}\left(z \times\left(y-y_{0}\right)\right)
$$

So, for finite $h$

$$
A_{n}(h)=O\left(\exp (-h) h^{n+1}\right) \begin{cases}=0, & \text { if } h=0 \\ \rightarrow 0, & \text { as } h \rightarrow \infty \text { in } C^{p} \\ =O\left(h^{n+1}\right), & \text { as } n \rightarrow \infty \text { in } N^{p}\end{cases}
$$

and $A_{n}(h)$ is not defined if for some $j, 1 \leq j \leq p, n_{j}>0$ and Reh $h_{j}=-\infty$. In particular, for $n$ and $v$ in $N^{p}$ and $y_{0}=-\infty \times \operatorname{Re} z$,

$$
\left(\int_{y_{0}}^{y} \mathrm{~d} y\right)^{n} \phi(y) \exp \left(y^{\prime} z\right)=\sum_{\lambda}\left(\begin{array}{c}
-n \\
\lambda
\end{array}\right) \phi^{(\lambda)}(y) z^{-n-\lambda} \exp \left(y^{\prime} z\right)
$$

and

$$
\begin{aligned}
\left(\int_{y_{0}}^{y} \mathrm{~d} y\right)^{n}\left(-\partial_{y}\right)^{\gamma} \phi(y) \exp \left(y^{\prime} z\right) & =\exp \left(y^{\prime} z\right) \sum_{\lambda}(-)^{\lambda}\left(\begin{array}{c}
-n \\
\lambda
\end{array}\right) z^{-n-\lambda}\left(-\partial_{y}\right)^{\gamma+\lambda} \phi(y) \\
& =\exp \left(y^{\prime} z\right) \sum_{\lambda} z^{-1-\lambda}\left(-\partial_{y}\right)^{\gamma+\lambda} \phi(y) \text { if } n=1 .
\end{aligned}
$$

Further formulae may be obtained by transforming (2.12). For example, multiplying (2.12) by $\partial_{z}^{m}$ for $m$ in $N^{p}$,

$$
\begin{aligned}
\left(\int_{y_{0}}^{y} \mathrm{~d} y\right)^{n} \phi(y) \exp \left(y^{\prime} z\right) y^{m} & =\sum_{\lambda}\left(\begin{array}{c}
-n \\
\lambda
\end{array}\right) \phi^{(\lambda)}(y) \sum_{k} z^{-n-\lambda+m-2 k}(-n-\lambda)_{k} \exp \left(y^{\prime} z\right) \\
& =\phi(y) \exp \left(y^{\prime} z\right) z^{m-n} \sum_{j} z^{-j} c_{j m n}(y)
\end{aligned}
$$

where

$$
c_{j m n}(y)=\sum_{k}(-)^{k}\left(\begin{array}{c}
m \\
k
\end{array}\right)\left(\begin{array}{c}
n-1+j-k \\
j-k
\end{array}\right) H_{j-2 k}(y) /(j-2 k) !=c_{j}
$$

say. So, $c_{0}=1$ and if $p=1$ then

$$
\begin{aligned}
& c_{1}=n H_{1}, \\
& c_{2}=\left(\begin{array}{c}
n+1 \\
2
\end{array}\right) H_{2} / 2-m n, \\
& c_{3}=\left(\begin{array}{c}
n+2 \\
3
\end{array}\right) H_{3} / 3 !-m\left(\begin{array}{c}
n+1 \\
2
\end{array}\right) H_{1}, \\
& c_{4}=\left(\begin{array}{c}
n+3 \\
4
\end{array}\right) H_{4} / 4 !-m\left(\begin{array}{c}
n+2 \\
3
\end{array}\right) H_{2} / 2+\left(\begin{array}{c}
m \\
2
\end{array}\right)\left(\begin{array}{c}
n+1 \\
2
\end{array}\right),
\end{aligned}
$$

where $H_{r}=H_{r}(y)$. 
Note 2.4. Note that (2.12) and (2.13) remain good as asymptotic expansions in inverse powers of $z$ for $y_{0}$ finite if $\operatorname{sign}\left(y-y_{0}\right)=\operatorname{sign} z$. For, if $p=1$ and $h=z\left(y-y_{0}\right)$ then $z^{-n} \exp (y z)\left(1-A_{n}(h)\right)$ is exponentially small as $|z| \rightarrow \infty$ for fixed $y-y_{0} \neq 0$.

Example 2.3. Take $\phi_{1}(y)=y^{z}$ and $y_{0}$ finite. Set

$$
B_{v}(y, z)=\partial_{y}^{v} y^{z}=\prod_{j=1}^{p} \partial_{y_{j}}^{v_{j}} y_{j}^{z_{j}}=\prod_{j=1}^{p} B_{v_{j}}\left(y_{j}, z_{j}\right) .
$$

So, $B_{v}(y, z)=y^{z-v}(z)_{v}$ if $v \geq 0$ and if $n=-v>1$ then

$$
\begin{aligned}
B_{v}(y, z) & =y^{z+n} /(z+n)_{n}-\sum_{k=1}^{n} y_{0}^{z+k}\left(y-y_{0}\right)^{n-k} /\left\{(z+k)_{k}(n-k) !\right\} \\
\partial_{y}^{v} y^{z} \phi(y) & =\sum_{\lambda}\left(\begin{array}{l}
v \\
\lambda
\end{array}\right) B_{v-\lambda}(y, z) \phi^{(\lambda)}(y) .
\end{aligned}
$$

Now fix $v_{0}$ in $N^{p}$ and take $\phi_{2}(y)=\left(-\partial_{y}\right)^{v_{0}} \phi(y)$. Then

$$
\partial_{y}^{v}\left\{y^{z}\left(-\partial_{y}\right)^{v_{0}} \phi(y)\right\}=\sum_{\lambda}(-)^{\lambda}\left(\begin{array}{l}
v \\
\lambda
\end{array}\right) B_{v-\lambda}(y, z) H_{v_{0}+\lambda}(y) \phi(y)
$$

for $H_{\lambda}(y)$ of (1.2).

\section{Multivariate ReCtangle Probabilities}

Theorem 3.1 provides various Taylor series expansions for $P(a<Y<b)$ in powers of $b-a$, for a random variable $Y$ in $R^{p}$.

Theorem 3.1. Suppose $Y$ is a random variable in $R^{p}$ with density $\phi$ and distribution $\Phi$. For $a<b$ in $R^{p}$,

$$
P(a<Y<b)=(-)^{q} G\left(y, y-y_{0}\right)
$$

where

$$
q=\sum_{j=1}^{p} I\left(y_{j}<y_{0 j}\right), a=\min \left(y_{0}, y\right), b=\max \left(y_{0}, y\right)
$$

and

$$
G(y, h)=\phi(y) \sum_{\lambda} H_{\lambda}(y) h^{\lambda+1} /(\lambda+1) !
$$

for $H_{\lambda}(y)$ of (1.2).

Proof. The Taylor series expansion in (3.1) follows by (2.9) with $n=1$.

There are $2^{p}$ choices for $\left(y_{0}\right)$ since $\left(y_{0 j}, y_{j}\right)=\left(a_{j}, b_{j}\right)$ or $\left(b_{j}, a_{j}\right)$. Corollary 3.1 considers some of these choices. 
Corollary 3.1. If $\left(y_{0}, y\right)=(a, b)$ then $(3.1)$ gives

$$
P(a<Y<b)=\phi(b) \sum_{\lambda} H_{\lambda}(b)(b-a)^{\lambda+1} /(\lambda+1) ! .
$$

If $\left(y_{0}, y\right)=(b, a)$ then $(3.1)$ gives

$$
P(a<Y<b)=\phi(a) \sum_{\lambda} H_{\lambda}(a)(a-b)^{\lambda+1} /(\lambda+1) ! .
$$

Note 3.1. If $Y$ is symmetric, that is if $P(Y<y)=P(-Y<y)$ or $\phi(-y)=\phi(y)$, then $(-)^{\lambda} H_{\lambda}(-y)=H_{\lambda}(y)$ and for $|\lambda|$ odd $H_{\lambda}(0)=0$ which makes expansions in $\left\{H_{\lambda}(0)\right\}$ attractive using

$$
\sum_{\lambda} c(\lambda) H_{\lambda}(0)=\sum_{r=0}^{\infty} \sum_{\lambda}^{2 r} c(\lambda) H_{\lambda}(0) .
$$

If also $Y \sim N_{p}(0, V)$ then by Theorem A.2 in the appendix

$$
H_{\lambda}(0)=E(i W)^{\lambda}
$$

where $W \sim N_{p}\left(0, V^{-1}\right)$ and $i=\sqrt{-1}$.

Corollaries 3.2 and 3.3 considers a rectangular decomposition, where $a$ and $b$ satisfy

$$
a_{j} b_{j}=0
$$

for $1 \leq j \leq p$. Any rectangle in $R^{p}$ can be decomposed as sums and differences of $2^{p}$ rectangles satisfying (3.5), since for $p=1,[a, b]=[a, 0)+[0, b]$ if $a<0<b,[a, b]=[0, b]-(0, a)$ if $0<a<b$ and $[a, b]=[a, 0]-(b, 0]$ if $a<b<0$. The corollaries use this fact to establish representations for $P(a<Y<b)$.

Corollary 3.2. Suppose (3.5) holds. Then, taking $y=0$ and $y_{0}=a_{j}$ if $a_{j} \neq 0$ and $y_{0}=b_{j}$ if $b_{j} \neq 0$, (3.1) gives

$$
P(a<Y<b)=(-)^{p-Q} G\left(0, y_{0}\right)=(-)^{p-Q} \phi(0) \sum_{\lambda} H_{\lambda}(0) y_{0}^{\lambda+1} /(\lambda+1) !
$$

where $Q=\sum\left\{I\left(a_{j}>0\right)+I\left(b_{j}>0\right)\right\}$.

Corollary 3.3. Under the assumptions of Corollary 3.2,

$$
P(a<Y<b)=\prod_{j=1}^{p}\left(B_{j}-A_{j}\right)
$$

with $X_{1} \ldots X_{p}$ replaced by $F(x)=G(0, x)$, where $x_{j}=a_{j}$ for $X_{j}=A_{j}$ and $x_{j}=b_{j}$ for $X_{j}=B_{j}$.

Proof. First suppose $0<a<b$. Then $[a, b]=\prod_{1}^{p}\left\{\left[0, b_{j}\right]-\left[0, a_{j}\right)\right\}=\prod_{1}^{p}\left(\overline{B_{j}}-\overline{A_{j}}\right)$ say, so $\mathrm{P}(a<Y<b)=$ $\sum(-)^{R} P(0<Y<B)$ summed over the $2^{p}$ possibilities $B_{j}=a_{j}$ or $b_{j}$, where $R$ is the number of $\left\{a_{j}\right\}$ in $B$. So, (3.6) holds with $Q=p$ and so (3.7) holds. Now suppose $a_{1}<0<b_{1}$ and $0<a_{j}<b_{j}$ for $2 \leq j \leq p$. Then

$$
[a, b]=\left(\left[a_{1}, 0\right]+\left(0, b_{1}\right]\right) \times \prod_{2}^{p}\left\{\left[0, b_{j}\right)-\left[0, a_{j}\right)\right\}
$$


so $P(a<Y<b)=\sum(-)^{S} P(A<Y<B)$ summed over the $2^{p}$ possibilities $\left(A_{1}, B_{1}\right)=\left(a_{1}, 0\right)$ or $\left(0, b_{1}\right)$ and $\left(A_{j}, B_{j}\right)=\left(0, a_{j}\right)$ or $\left(0, b_{j}\right)$ for $2 \leq j \leq p$, and $S$ is the number of $\left(A_{j}, B_{j}\right)=\left(0, a_{j}\right)$ for $j \geq 2$. The choice $\left(A_{1}, B_{1}\right)=\left(0, b_{1}\right)$ gives $S=R$ and the same contribution as for $0<a<b$. A typical term with $\left(A_{1}, B_{1}\right)=\left(a_{1}, 0\right)$ is

$$
\left(a_{1}, 0\right) \times \prod_{2}^{S+1}\left(0, a_{j}\right) \times \prod_{S+2}^{p}\left(0, b_{j}\right)
$$

This gives $y_{0 j}=a_{j}$ for $j \leq S+1$ and $y_{0 j}=b_{j}$ for $j>S+1$, so $p-Q=1$ in (3.6). This change in sign cancels with the change in sign from replacing $+\left[a_{1}, 0\right)$ in the previous case by $-\left[0, a_{1}\right)$. So, (3.7) still holds. A similar cancelation of sign change takes place if $a_{1}<b_{1}<0$. (For example, if $p=1$ then $P(a<Y<b)=P(a<Y<$ $0)-P(b<Y<0), P(a<Y<0)=(-)^{1-Q} F(a)$ with $Q=0$ and $P(b<Y<0)=(-)^{1-Q} F(b)$ with $Q=0$. $)$ So, (3.6) holds for $a_{1}<b_{1}$. Similarly, it holds for $a_{j}<b_{j}, 2 \leq j \leq p$. This completes the proof of (3.7).

Example 3.1. For $p=1,2,3,(3.7)$ reduces to

$$
\begin{gathered}
P(a<Y<b)=F(b)-F(a), \\
P(a<Y<b)=F(b)-F\left(b_{1}, a_{2}\right)-F\left(a_{1}, b_{2}\right)+F(a),
\end{gathered}
$$

and

$$
\begin{aligned}
P(a<Y<b)= & F(b)-F\left(b_{1}, b_{2}, a_{3}\right)-F\left(b_{1}, a_{2}, a_{3}\right)-F\left(a_{1}, b_{2}, b_{3}\right)+F\left(a_{1}, a_{2}, b_{3}\right) \\
& +F\left(a_{1}, b_{2}, a_{3}\right)+F\left(b_{1}, a_{2}, a_{3}\right)-F(a)
\end{aligned}
$$

respectively.

\section{Multivariate nORMal Probabilities}

Theorem 4.1 provides expansions for $P(a<Y<b), Y \sim N_{p}(0, V)$ in terms of multivariate Hermite polynomials.

Theorem 4.1. Suppose $Y \sim N_{p}(0, V)$ with det $V \neq 0$, density $\phi$ and distribution $\Phi$. Fix $z$ in $R^{p}$ with $z_{1} \ldots z_{p} \neq 0$ and set $\mu=V z$. Then

$$
P(a<Y<b)=(-)^{q} \phi(y-\mu) \sum_{\lambda} H_{\lambda}(y) z^{-1-\lambda} A_{1+\lambda}(h)=(-)^{q} F\left(y, y_{0}, z\right)
$$

say for $q$ of (3.2), for $A_{n}$ of (2.10) and for $h=z \times\left(y-y_{0}\right)$ in $(-\infty, \infty]$, where $a+\mu=\min \left(y_{0}, y\right)$ and $b+\mu=\max \left(y_{0}, y\right)$. Furthermore, $\left\{H_{\lambda}(y)\right\}$ are the multivariate Hermite polynomials given explicitly in the appendix.

Proof. Note $\phi(y) \exp \left(y^{\prime} z\right)=\phi(y-\mu) \alpha$, where $\alpha=\exp \left(\mu^{\prime} V^{-1} \mu\right)=\exp \left(z^{\prime} V z\right)$. Dividing (2.11) by $\alpha$ gives for $n$ in $Z^{p}$ and $y_{0}-\mu=y-\mu_{0}$

$$
\begin{aligned}
\left(\int_{y_{0}}^{y} \mathrm{~d} y\right)^{n} \phi(y-\mu) & =\left(-\int_{\mu_{0}}^{\mu} \mathrm{d} \mu\right)^{n} \phi(\mu-y)=\left(\int_{\mu}^{\mu_{0}} \mathrm{~d} \mu\right)^{n} \phi(\mu-y) \\
& =\phi(y-\mu) \sum_{\lambda}\left(\begin{array}{c}
n-1+\lambda \\
\lambda
\end{array}\right) H_{\lambda}(y) z^{-n-\lambda} A_{n+\lambda}(h) .
\end{aligned}
$$

Set $n=1$ to obtain the result. 
As in Section 3 we can choose $\left(y_{0}, y\right)$ to satisfy $a+\mu=\min \left(y_{0}, y\right)$ and $b+\mu=\max \left(y_{0}, y\right)$ in $2^{p}$ ways so that (4.1) gives $2^{p}$ expansions. Two examples of this are illustrated in Corollary 4.1.

Corollary 4.1. If $\left(y_{0}, y\right)=(a+\mu, b+\mu)$ then (4.1) gives

$$
P(a<Y<b)=\phi(b) \sum_{\lambda} H_{\lambda}(b+V z) z^{-1-\lambda} A_{1+\lambda}((b-a) \times z) .
$$

If $\left(y_{0}, y\right)=(b+\mu, a+\mu)$ then (4.1) gives

$$
P(a<Y<b)=(-)^{q} \phi(a) \sum_{\lambda} H_{\lambda}(a+V z) z^{-1-\lambda} A_{1+\lambda}((a-b) \times z) .
$$

We now apply the rectangle decomposition of Section 3 with $a$ and $b$ replaced by $a+\mu$ and $b+\mu$, respectively. That is, we decompose $[a, b]$ into sums and differences of $2^{p}$ rectangles $[a, b]$ such that $\left(a_{j}+\mu_{j}\right)\left(b_{j}+\mu_{j}\right)=0$ for $1 \leq j \leq p$, and see how Corollaries 3.2 and 3.3 change.

Corollary 4.2. Suppose $\left(a_{j}+\mu_{j}\right)\left(b_{j}+\mu_{j}\right)=0$ for $1 \leq j \leq p$. Taking $y=0$ and $y_{0 j}=a_{j}+\mu_{j}$ if $a_{j}+\mu_{j} \neq 0$ and $y_{0 j}=b_{j}+\mu_{j}$ if $b_{j}+\mu_{j} \neq 0$, we see (4.1) gives

$$
P(a<Y<b)=(-)^{Q} F\left(0, y_{0}, z\right),
$$

where

$$
Q=\sum_{j=1}^{p}\left\{I\left(a_{j}+\mu_{j}>0\right)+I\left(b_{j}+\mu_{j}>0\right)\right\}
$$

Corollary 4.3. Under the assumptions of Corollary 4.2,

$$
\begin{aligned}
P(a<Y<b) & =(-)^{q} \phi(\mu) \sum_{\lambda} H_{\lambda}(0) z^{-1-\lambda} A_{1+\lambda}(a, b) \\
& =(-)^{q} \phi(\mu) \sum_{r=0}^{\infty}(-)^{r} \sum_{\lambda}^{2 r} E W^{\lambda} z^{-1-\lambda} A_{1+\lambda}(a, b),
\end{aligned}
$$

where $W \sim N\left(0, V^{-1}\right)$ and

$$
A_{1+\lambda}(a, b)=\prod_{j=1}^{p}\left(B_{j}-A_{j}\right)
$$

at $X_{1} \ldots X_{p}=A_{1+\lambda}(-z \times(x+\mu))$ for $x_{j}=a_{j}$ if $X_{j}=A_{j}$ and $x_{j}=b_{j}$ if $X_{j}=B_{j}$.

Proof. Note from (4.1) that

$$
F(x)=(-)^{q} F(0, x+V z, z)=(-)^{q} \phi(\mu) \sum_{r=0}^{\infty}(-)^{r} \sum_{\lambda}^{2 r} E W^{\lambda} z^{-1-\lambda} A_{1+\lambda}(h)
$$

provided $h=-z \times(x+\mu)$ is in $(-\infty, \infty]$. So, (3.7) can be written in the form (4.2).

Example 4.1. For $p=1, A_{1+\lambda}(a, b)=A_{1+\lambda}\left(-b z-V z^{2}\right)-A_{1+\lambda}\left(-a z-V z^{2}\right)$. For $p=2, A_{1+\lambda}(a, b)=$ $\sum_{1}^{4}(-)^{j} A_{1+\lambda}\left(-z \times\left(x_{j}+\mu\right)\right)$, where $x_{2}=a, x_{4}=b, x_{1}=\left(b_{1}, a_{2}\right)^{\prime}$ and $x_{3}=\left(a_{1}, b_{2}\right)^{\prime}$. 
Note 4.1. If $b-a$ is small, it may be more accurate to use one of the $2^{p}$ expansions (3.1) or (4.1) rather than (3.7) with $F(x)=G(0, x)$ or $(4.2)$.

The expansion (4.1) and derived expressions such as (4.2) are remarkable in that $z$ is any element in $R^{p}$ with non-zero components. The proviso to this is that $h>-\infty$ in $A_{1+\lambda}(h)$. So, in (4.2) $z_{j}>0$ if $a_{j}=-\infty$ and $z_{j}<0$ if $b_{j}=\infty$. See Corollary 4.4 for examples. Some further particular cases of (4.1) are considered by Corollary 4.5.

Corollary 4.4. Taking $a=-\infty$ in (4.2), for $z>0$

$$
\Phi(b)=P(Y<b)=(-)^{q} \phi(\mu) \sum_{\lambda} H_{\lambda}(0) z^{-1-\lambda} A_{1+\lambda}(-\infty, b),
$$

where

$$
\begin{aligned}
A_{1+\lambda}(-\infty, b) & =A_{1+\lambda}\left(-b z-V z^{2}\right)-1 \text { if } p=1, \\
& =1+A_{1+\lambda}(-z \times(b+\mu))-\sum_{j=1}^{2} A_{1+\lambda}\left(-z_{j}\left(b_{j}+\mu_{j}\right)\right) \text { if } p=2
\end{aligned}
$$

and so on, while for $z<0$

$$
\Phi(-a)=P(a<Y)=(-)^{q} \phi(\mu) \sum_{\lambda} H_{\lambda}(0) z^{-1-\lambda} A_{1+\lambda}(a, \infty),
$$

where $A_{1+\lambda}(a, \infty)=1-A_{1+\lambda}\left(-a z-V z^{2}\right)$ if $p=1, A_{1+\lambda}(a, \infty)$ is equal to the right hand side of (4.3) at $b=a$ if $p=2$, and so on. In particular, for $p=V=1$ we have by (3.4)

$$
\Phi(x)=\phi(x) z^{-1} \sum_{r=0}^{\infty}\left(-z^{2}\right)^{-r} c_{r} A_{1+2 r}^{*},
$$

where

$$
c_{r}=E(N(0,1))^{2 r}=(2 r) ! 2^{-r} r !=1.3 \ldots(2 r-1)
$$

and

$$
\begin{aligned}
A_{n}^{*} & = \begin{cases}1-A_{n}\left(-x z-z^{2}\right), & \text { if } z>0, \\
A_{n}\left(x z-z^{2}\right)-1, & \text { if } z<0\end{cases} \\
& =\left\{1-A_{n}\left(-x|z|-z^{2}\right)\right\} \operatorname{sign} z,
\end{aligned}
$$

so the right hand side of (4.4) is symmetric about $z=0$. For $z=-x$ this gives the well known expansion

$$
\Phi(x)=\phi(x)|x|^{-1} \sum_{r=0}^{\infty}\left(-x^{2}\right)^{-r} c_{r}
$$

for $x<0$.

Note 4.2. The relative error in (4.6) is $\sim 10 \%$ at $x=-3$ and $\sim 1 \%$ at $x=-6$ if we truncate when

$$
\mid(r+1) \text { st term }|>\theta| r \text { th term } \mid \text {, where } \theta=1 \text { or } 0.5 \text {. }
$$

Table 1 gives the relative error of (4.4) for selected values of $\Phi(x)$ and $z+x$ truncating in the same way. The $x$ values are taken from Table 1.2 of [9]. The values of $\Phi(x)$ were computed using NAG. 
TABLE 1. Relative error of (4.4) truncating as in (4.7).

\begin{tabular}{|c|c|c|c|c|c|c|}
\hline & & & $\theta=1$ & & $\theta=0.5$ & \\
\hline$x$ & $\Phi(x)$ & $z+x$ & Relative error & $r$ & Relative error & $r$ \\
\hline-1.28155 & 0.1 & -0.1 & $-53 \%$ & 1 & $-53 \%$ & 1 \\
\hline & & 0 & $-46 \%$ & 1 & $-46 \%$ & 1 \\
\hline & & 0.1 & $-48 \%$ & 1 & $-48 \%$ & 1 \\
\hline-2.32635 & 0.01 & -0.1 & $35 \%$ & 2 & $17 \%$ & 1 \\
\hline & & 0 & $-6 \%$ & 3 & $-7 \%$ & 1 \\
\hline & & 0.1 & $-30 \%$ & 3 & $-31 \%$ & 1 \\
\hline-3.09023 & 0.001 & -0.1 & $35 \%$ & 4 & $36 \%$ & 2 \\
\hline & & 0 & $-0.66 \%$ & 5 & $1.1 \%$ & 2 \\
\hline & & 0.1 & $-34 \%$ & 5 & $-34 \%$ & 3 \\
\hline-3.71902 & 0.0001 & -0.1 & $40 \%$ & 7 & $39 \%$ & 3 \\
\hline & & 0 & $-0.0075 \%$ & 7 & $-0.19 \%$ & 3 \\
\hline & & 0.1 & $-40 \%$ & 7 & $-40 \%$ & 4 \\
\hline-4.74342 & $10^{-6}$ & -0.1 & $50 \%$ & 11 & $50 \%$ & 5 \\
\hline & & 0 & $-0.0001 \%$ & 11 & $0.0003 \%$ & 6 \\
\hline & & 0.1 & $-50 \%$ & 12 & $-50 \%$ & 6 \\
\hline-5.99781 & $10^{-9}$ & -0.1 & $62 \%$ & 17 & $62 \%$ & 9 \\
\hline & & 0 & $0.000001 \%$ & 18 & $-0.00001 \%$ & 9 \\
\hline & & 0.1 & $-62 \%$ & 19 & $-62 \%$ & 9 \\
\hline
\end{tabular}

Corollary 4.5. Putting $y_{0}=-\infty \times z$ and $x=y-\mu$ in (4.1) gives

$$
P((Y-x) \times \operatorname{sign} z<0)=(-)^{q} \phi(x) \sum_{\lambda} H_{\lambda}(x+V z) z^{-1-\lambda},
$$

where $q=\sum_{j=1}^{p} I\left(z_{j}<0\right)$. In particular,

$$
\Phi(x)=P(Y<x)=\phi(x) \sum_{\lambda} H_{\lambda}(x+V z) z^{-1-\lambda} \text { if } z>0
$$

and since $-Y$ and $Y$ have the same distribution,

$$
\Phi(-x)=P(Y>x)=(-)^{q} \phi(x) \sum_{\lambda} H_{\lambda}(x+V z) z^{-1-\lambda} \text { if } z<0 .
$$

These can also be written as

$$
\Phi(y-V z) / \phi(y-V z)=\sum_{\lambda} H_{\lambda}(y) z^{-1-\lambda} \text { if } z>0
$$

and

$$
\Phi(-y+V z) / \phi(-y+V z)=(-)^{q} \sum_{\lambda} H_{\lambda}(y) z^{-1-\lambda} \text { if } z<0,
$$


respectively. Putting $z=-V^{-1} x$ in (4.8) gives

$$
(-)^{q} P((Y-x) \times \operatorname{sign} z<0)=\phi(x) \sum_{\lambda} H_{\lambda}(0) z^{-1-\lambda}=\phi(x) \sum_{r=0}^{\infty}(-)^{r} \sum_{\lambda}^{2 r} E W^{\lambda} z^{-1-\lambda}
$$

by (3.4). In particular, if $z=V^{-1} \mu>0$,

$$
\Phi(-\mu)=\phi(\mu) \sum_{\lambda} H_{\lambda}(0) z^{-1-\lambda}
$$

an expansion given by [11], while if $z=V^{-1} \mu<0$,

$$
\Phi(\mu)=(-)^{q} \phi(\mu) \sum_{\lambda} H_{\lambda}(0) z^{-1-\lambda} .
$$

All of the expansions in Corollary 4.5 reduce to (4.6) if $p=V=1$. The expansions with $y=0$ can be used to give upper and lower bounds: see Savage (1962) for some examples using the terms $r=0,1$ in (4.9).

Note 4.3. Suppose that

$$
z_{j} \begin{cases}>0, & \text { for } j \text { in } J \\ <0, & \text { for } j \text { in } K\end{cases}
$$

where $J \cup K=\{1, \ldots, p\}$. Set

$$
\left(Y_{j}^{*}, x_{j}^{*}, z_{j}^{*}\right)= \begin{cases}\left(Y_{j}, x_{j}, z_{j}\right), & \text { for } j \text { in } J \\ -\left(Y_{j}, x_{j}, z_{j}\right), & \text { for } j \text { in } K\end{cases}
$$

and $V^{*}=\operatorname{cov} Y^{*}$. Then the left hand side of (4.8) is $P\left(Y^{*}<x^{*}\right),(V z)^{*}=V^{*} z^{*}, \phi_{V}=\phi$ satisfies $\phi_{V}(x)=$ $\phi_{V^{*}}\left(x^{*}\right)$ and $H_{\lambda}(x, V)=H_{\lambda}(x)$ satisfies $(-)^{\lambda^{*}} H_{\lambda}\left(x^{*}, V^{*}\right)=H_{\lambda}(x, V)$, where $\lambda^{*}=\sum_{j=1}^{p} \lambda_{j} I\left(z_{j}<0\right)$. So, (4.8) gives: for $z^{*}>0$

$$
P\left(Y^{*}<x^{*}\right)=\phi_{V^{*}}\left(x^{*}\right) \sum_{\lambda} H_{\lambda}\left(x^{*}+V^{*} z^{*}, V^{*}\right)\left(z^{*}\right)^{-1-\lambda}
$$

which is just the ${ }^{*}$ version of (4.8) for $z>0$. Conversely, we could have obtained (4.8) from the case $z>0$.

Steck [13] commented that the condition $z>0$ in (4.10) "appears to be a severe one". We now show how to remove this condition in order to find an expansion for $\Phi(x)$ for almost any $x$ in $R^{p}$.

Note 4.4. Suppose $z=-V^{-1} x$ satisfies $z_{1} \ldots z_{p} \neq 0$. Define $J, K$ by (4.11). Let $q$ be the number of elements in $K$. Decompose $(-\infty, x)$ as $\prod_{j \in J}\left(-\infty, x_{j}\right) \times \prod_{k \in K}\left(-\infty, x_{k}\right)$. Then

$$
\Phi(x)=P(Y<x)=C \prod_{k \in K}\left(B_{k}-A_{k}\right),
$$

where

$$
C \prod_{k \in K} X_{j}=P\left(Y_{j}<x_{j} \text { for } j \in J, Y_{k}>x_{k}^{*} \text { for } k \in K\right)
$$


and $x_{k}^{*}=-\infty$ if $X_{k}=B_{k}$ and $x_{k}^{*}=x_{k}$ if $X_{k}=A_{k}$. The last of the $2^{q}$ terms in (4.12) is

$$
\begin{aligned}
(-)^{q} C \prod_{k \in K} A_{k} & =(-)^{q} P\left(Y_{j}<x_{j} \text { for } j \in J, Y_{k}>x_{k} \text { for } k \in K\right) \\
& =\phi(x) \sum_{\lambda} H_{\lambda}(0) z^{-1-\lambda} \text { by }(4.9) .
\end{aligned}
$$

The other $2^{q}-1$ terms are of the form $\pm P\left(Y_{j}^{*}>\right.$ or $\left.<x_{j}^{*}, 1 \leq j \leq r\right)$, where $r<p$ and $Y^{*} \sim N_{r}\left(0, V^{*}\right)$ say. These can all be dealt with in the same way. So, by repeated application of (4.9) the dimension is reduced from $p$ to 1 .

Example 4.2. Suppose $p=2$ and $z_{1}<0<z_{2}$. Then $\Phi(x)=P\left(Y_{2}<x_{2}\right)-P\left(Y_{1}>x_{1}, Y_{2}<x_{2}\right)$ is the sum of $\Phi_{1}\left(x_{2} V_{22}^{-1 / 2}\right)$ and the right hand side of $(4.9)$, where $\Phi_{1}$ is $\Phi$ for $N(0,1)$.

Example 4.3. Suppose $p=2$ and $z<0$. Then $\Phi(x)$ is the sum of $1-P_{1}-P_{2}$ and the right hand side of (4.9), where $P_{j}=P\left(Y_{j}>x_{j}\right)=\Phi_{1}\left(-x_{j} V_{j j}^{-1 / 2}\right)$.

Example 4.4. Suppose $p=3$ and $z_{1}<0<z_{2}, z_{3}$. Then $\Phi(x)$ is the sum of $P\left(Y_{2}<x_{2}, Y_{3}<x_{3}\right)$ and the right hand side of (4.9).

Example 4.5. Suppose $p=3$ and $z_{1}, z_{2}<0<z_{3}$. Then $\Phi(x)$ is the sum of $P\left(Y_{3}<x_{3}\right)-\sum_{1}^{2} P_{j}$ and the right hand side of (4.9), where $P_{j}=P\left(Y_{j}>x_{j}, Y_{3}<x_{3}\right)$.

We end this section with some explicit formulas for $\partial_{y}^{-n} \phi(y)$ and $\partial_{y}^{n} \Phi(y)$.

Example 4.6. Suppose $p=V=1$. Then

$$
\begin{aligned}
\int_{y_{0}}^{y}(y-x) \phi(x) \mathrm{d} x & =\left(\int_{y_{0}}^{y} \mathrm{~d} y\right)^{2} \phi(y) \\
& =\phi(y)-\phi\left(y_{0}\right)+y\left\{\Phi(y)-\Phi\left(y_{0}\right)\right\} \\
& =\phi(y)+y \Phi(y) \text { if } y_{0}=-\infty
\end{aligned}
$$

For, the left and right hand sides are equal at $y=y_{0}$ and so are their partial derivatives with respect to $y$. Similarly,

$$
\begin{aligned}
2\left(\int_{y_{0}}^{y} \mathrm{~d} y\right)^{3} \phi(y) & =y \phi(y)-2 y \phi\left(y_{0}\right)+y_{0} \phi\left(y_{0}\right)+\left(1+y^{2}\right)\left\{\Phi(y)-\Phi\left(y_{0}\right)\right\} \\
& =y \phi(y)+\left(1+y^{2}\right) \Phi(y) \text { if } y_{0}=-\infty .
\end{aligned}
$$

More generally, for $r \geq 0$

$$
\begin{aligned}
r !\left(\int_{y_{0}}^{y} \mathrm{~d} y\right)^{r+1} \phi(y)= & a_{r-1}(y)\left\{\phi(y)-\phi\left(y_{0}\right)\right\}+b_{r-1}\left(y, y_{0}\right) \phi\left(y_{0}\right) \\
& +H_{r}^{+}(y)\left\{\Phi(y)-\Phi\left(y_{0}\right)\right\} \\
= & a_{r-1}(y) \phi(y)+H_{r}^{+}(y) \Phi(y) \text { if } y_{0}=-\infty,
\end{aligned}
$$

where $H_{r}^{+}(y)$ is $H_{r}(y)$ with all signs made positive:

$$
H_{r}^{+}(y)=\sum\left\{y^{r-2 j}(r)_{2 j} 2^{-j} / j !: 0 \leq j \leq r / 2\right\}
$$


and $a_{j}$ and $b_{j}$ are polynomials of degree $j$. For, differentiating (4.13) gives

$$
r H_{r-1}^{+}(y)=\partial_{y} H_{r}^{+}(y), r a_{r-2}(y)=\left(\partial_{y}-y\right) a_{r-1}(y)+H_{r}^{+}(y),
$$

and

$$
-r a_{r-2}(y)+r b_{r-2}\left(y, y_{0}\right)=\partial_{y}\left(-a_{r-1}(y)+b_{r-1}\left(y, y_{0}\right)\right) .
$$

Now apply induction. The last equation gives the recursive formula for $b_{r}$ :

$$
b_{r}\left(y, y_{0}\right)=(r+1) \int_{y_{0}}^{y}\left\{b_{r-1}\left(y, y_{0}\right)-a_{r-1}(y)\right\} \mathrm{d} y+a_{r}(y)-a_{r}\left(y_{0}\right) .
$$

It would be interesting to know if (4.13) can be extended to $p>1$.

Example 4.7. Set $\Phi_{V}(y)=\Phi(y), \phi_{V}(y)=\phi(y)$, partition $y^{\prime}=\left(y^{\prime}, y_{0}^{\prime}\right)$, and similarly $V$ is a $2 \times 2$ block matrix. Set $M_{12}=V_{12} V_{22}^{-1}$ and $V_{1 \cdot 2}=V_{11}-V_{12} V_{22}^{-1} V_{21}$. Since $Y_{1} / Y_{2} \sim \mathcal{N}\left(M_{12} Y_{2}, V_{1 \cdot 2}\right)$,

$$
\Phi_{V}(y)=\int^{y_{2}} \mathrm{~d} y_{2} \phi_{V_{22}}\left(y_{2}\right) \Phi_{V_{1 \cdot 2}}\left(y_{1}-M_{12} y_{2}\right) .
$$

Now suppose $p=2$. Then

$$
\begin{gathered}
\partial_{y_{2}} \Phi_{V}(y)=\phi_{V_{22}}\left(y_{2}\right) \Phi_{V_{1 \cdot 2}}\left(y_{1}-M_{12} y_{2}\right) \\
\partial_{y_{2}}^{2} \Phi_{V}(y)=\phi_{V_{22}}^{\prime}\left(y_{2}\right) \Phi_{V_{1 \cdot 2}}\left(y_{1}-M_{12} y_{2}\right)-M_{12} \phi_{V_{22}}\left(y_{2}\right) \phi_{V_{1 \cdot 2}}\left(y_{1}-M_{12} y_{2}\right),
\end{gathered}
$$

and so on.

Acknowledgements. The authors would like to thank the Editors-in-Chief, the Associate Editor and the two referees for carefully reading the paper and for their comments which greatly improved the paper.

\section{A. The Multivariate HeRmite POLYNOMials}

Suppose $Y \sim N_{p}(0, V)$, and $\operatorname{det} V \neq 0$. Then $\phi(y)=(2 \pi)^{-p / 2}(\operatorname{det} V)^{-1 / 2} \exp \left(-y^{\prime} V^{-1} y / 2\right)$ and $\left\{H_{\lambda}(y)\right\}$ are the multivariate Hermite polynomials. They can be obtained iteratively from

$$
H_{\lambda+e_{j}}(y)=\left(x_{j}+\partial_{y_{j}}\right) H_{\lambda}(y), H_{0}(y)=1,
$$

where $e_{j}$ is the $j$ th unit vector in $R^{p}$ and $x=V^{-1} y$. However, an explicit form is easily given by using the dual form

$$
\begin{aligned}
H^{\alpha_{1} \ldots \alpha_{r}}(y) & =\phi(y)^{-1} D_{\alpha_{1}} \ldots D_{\alpha_{r}} \phi(y) \text { for } D=-\partial / \partial y \\
& =H_{\lambda}(y) \text { for }|\lambda|=r \text { and } \lambda_{j} \text { the number of } j \text { 's in }\left\{\alpha_{1}, \ldots \alpha_{r}\right\}
\end{aligned}
$$

Set $x=V^{-1} y$ and $\left(V^{i j}\right)=V^{-1}$. By (A.1),

$$
H^{\alpha_{1} \ldots \alpha_{r}}(y)=\left(x_{\alpha_{r}}+D_{\alpha_{r}}\right) H^{\alpha_{1} \ldots \alpha_{r-1}}(y),
$$


SO

$$
\begin{aligned}
& H^{\alpha_{1}}(y)=x_{\alpha_{1}}, H^{\alpha_{1} \alpha_{2}}(y)=x_{\alpha_{1}} x_{\alpha_{2}}-V^{\alpha_{1} \alpha_{2}} \\
& H^{\alpha_{1} \alpha_{2} \alpha_{3}}(y)=x_{\alpha_{1}} x_{\alpha_{2}} x_{\alpha_{3}}-\sum^{3} x_{\alpha_{1}} V^{\alpha_{2} \alpha_{3}} \\
& H^{\alpha_{1} \ldots \alpha_{4}}(y)=x_{\alpha_{1}} \ldots x_{\alpha_{4}}-\sum^{6} x_{\alpha_{1}} x_{\alpha_{2}} V^{\alpha_{3} \alpha_{4}}+\sum^{3} V^{\alpha_{1} \alpha_{2}} V^{\alpha_{3} \alpha_{4}},
\end{aligned}
$$

where $\sum^{j}$ sums over all $j$ permutations of $\alpha_{1} \ldots \alpha_{r}$ giving distinct terms. Comparing these with the univariate Hermite polynomials:

$$
H_{r}(x)=\sum\left\{(-)^{j-1} R(r, j) x^{r-2 j}: 0 \leq j \leq r / 2\right\}
$$

where $R(r, j)=(r)_{2 j} 2^{-j} / j$ !, we see the following result.

Theorem A.1. With the notation as above,

$$
H^{\alpha_{1} \ldots \alpha_{r}}(y)=\sum\left\{(-)^{j-1} \sum^{R(r, j)} x_{\alpha_{1}} \ldots x_{\alpha_{r-2 j}} V^{\beta_{1} \beta_{2}} \ldots V^{\beta_{2 j-1} \beta_{2 j}}: 0 \leq j \leq r / 2\right\}
$$

where $\beta_{k}=\alpha_{r-2 j+k}$. Setting $y=0$ gives

$$
(-)^{r} H^{\alpha_{1} \ldots \alpha_{2 r}}(0)=\sum^{c_{r}} V^{\alpha_{1} \alpha_{2}} \ldots V^{\alpha_{2 r-1} \alpha_{2 r}}
$$

for $R(2 r, r)=c_{r}$ of $(4.5)$.

Barndorff-Nielsen and Pederson [1] gave expressions for $H^{\alpha_{1} \ldots \alpha_{r}}(y)$ for $p=2$ and $r \leq 6$ without recognising the general form (A.2). For a formal proof of (A.2) see Withers [16].

Theorem A.2. If $W \sim N_{p}\left(0, V^{-1}\right)$ then $H_{\lambda}(y)=E(i W)^{\lambda} \exp \left(i y^{\prime} W\right), H_{\lambda}(0)=E(i W)^{\lambda}, H^{\alpha_{1} \ldots \alpha_{r}}(y)=$ $E\left(i W_{\alpha_{1}}\right) \ldots\left(i W_{\alpha_{r}}\right) \exp \left(i y^{\prime} W\right)$ and $H^{\alpha_{1} \ldots \alpha_{r}}(0)=i^{r} E W_{\alpha_{1}} \ldots W_{\alpha_{r}}$.

Proof. Simply note that $\phi(0)^{-1} \phi(y)=E \exp \left(i y^{\prime} W\right)$ for $i=\sqrt{-1}$.

It follows from Theorem A.2 that $E W_{\alpha_{1}} \ldots W_{\alpha_{2 r}}$ is equal to the right hand side of (A.2), as given by Corollary 1.1 of Withers [17]. Some of Withers [17] results are duplicated in Holmquist [4].

Theorem A.3. Suppose $W \sim N_{p}\left(0, V^{-1}\right)$. Set $\mu=V z$. For $n \in R^{p}, \phi(y-\mu)^{-1} \partial_{y}^{n} \phi(y-\mu)=E(z-$ $i W)^{n} \exp \left(i y^{\prime} W\right)$. Specialising to $n$ in $N^{p}, H_{n}(\mu)=E(z-i W)^{n}$.

Proof. By (2.11) for $y_{0}=-\infty \times z$,

$$
\begin{aligned}
\phi(y-\mu)^{-1} \partial_{y}^{n} \phi(y-\mu) & =z^{n} \sum_{\lambda}\left(\begin{array}{c}
n \\
\lambda
\end{array}\right) H_{\lambda}(y)(-z)^{-\lambda} \\
& =z^{n} \sum_{\lambda}\left(\begin{array}{c}
n \\
\lambda
\end{array}\right) E\left(-i W \times z^{-1}\right)^{\lambda} \exp \left(i y^{\prime} W\right) \\
& =z^{n} E\left(1-i W \times z^{-1}\right)^{n} \exp \left(i y^{\prime} W\right) \\
& =E(z-i W)^{n} \exp \left(i y^{\prime} W\right) .
\end{aligned}
$$

The result follows. 
We now show how to convert a sum $\sum_{\lambda}^{r}$ to its dual.

Note A.5. Let $\sum_{\alpha}^{p, r}$ denote summation over $\alpha_{1}, \ldots, \alpha_{r}$ in $\{1, \ldots, p\}$. Then

$$
\sum_{\alpha}^{p, r} y_{\alpha_{1}} \ldots y_{\alpha_{r}}=\left(1^{\prime} y\right)^{r}=r ! \sum_{\lambda}^{r} y^{\lambda} / \lambda !
$$

So, we can write

$$
\begin{aligned}
& z^{n} \sum_{\lambda}\left(\begin{array}{l}
n \\
\lambda
\end{array}\right) H_{\lambda}(y)(-z)^{-\lambda} \\
= & z^{n} \sum_{r=0}^{\infty} \sum_{\lambda}^{r}(n)_{\lambda} H_{\lambda}(y)(-z)^{-\lambda} / \lambda ! \\
= & z^{n} \sum_{r=0}^{\infty}(r !)^{-1} \sum_{\alpha}^{p, r}(n)_{\lambda_{\alpha}} H^{\alpha_{1} \ldots \alpha_{r}}(y)\left(-z_{\alpha_{1}}\right)^{-1} \ldots\left(-z_{\alpha_{r}}\right)^{-1},
\end{aligned}
$$

where $\lambda_{\alpha}=e_{\alpha_{1}}+\ldots+e_{\alpha_{r}}$, and $e_{j}$ is as in (A.1). More generally,

$$
\sum_{\alpha}^{p, r} f(\alpha) y_{\alpha_{1}} \ldots y_{\alpha_{r}}=r ! \sum_{\lambda}^{r} \bar{f}(\lambda) y^{\lambda} / \lambda !
$$

if either $f(\alpha)=\bar{f}\left(\lambda_{\alpha}\right)$ or $f(\alpha)$ is symmetric and $\bar{f}(\lambda)=f\left(\alpha_{\lambda}\right)$, where $\alpha_{\lambda}=\left(1^{\lambda_{1}}, \ldots, p^{\lambda_{p}}\right)^{\prime}$ and $j^{\lambda j}=(j, \ldots, j)$ has dimension $\lambda_{j}$. For example, we can write $G$ in (3.3), (3.6) as

$$
\begin{aligned}
G(y, h) & =h^{1} \phi(y) \sum_{r=0}^{\infty} \sum_{\lambda}^{r} H_{\lambda}(y) h^{\lambda} /\left\{(\lambda+1)^{1} \lambda !\right\} \\
& =h^{1} \phi(y) \sum_{r=0}^{\infty}(r !)^{-1} \sum_{\alpha}^{p, r} H^{\alpha_{1} \ldots \alpha_{r}}(y) h_{\alpha_{1}} \ldots h_{\alpha_{r}}\left(\lambda_{\alpha}+1\right)^{-1}
\end{aligned}
$$

and Ruben's expansion, (4.10), as:

$$
\Phi(-\mu)=\phi(\mu) z^{-1} \sum_{r=0}^{\infty}(-)^{r}(r !)^{-1} \sum_{\alpha}^{p, 2 r} \lambda_{\alpha} ! E W_{\alpha_{1}} \ldots W_{\alpha_{2 r}} z_{\alpha_{1}}^{-1} \ldots z_{\alpha_{2 r}}^{-1}
$$

for $z=V^{-1} \mu>0$.

\section{REFERENCES}

[1] O. Barndorff-Nielsen and B.V. Pederson, The bivariate Hermite polynomials up to order six. Scand. J. Stat. 6 (1978) $127-128$.

[2] R.A. Fisher, Introduction of "Table of Hh functions", of Airey (1931), xxvi-xxxvii, Mathematical Tables, 2nd edition 1946, 3th edition 1951. British Association for the Advancement of Science, London (1931), Vol. 1,

[3] C.R. Goodall and K.V. Mardia, A geometric derivation of the shape density. Adv. Appl. Prob. 23 (1991) 496-514.

[4] B. Holmquist, Moments and cumulants of the multivariate normal distribution. Stoch. Anal. Appl. 6 (1988) $273-278$.

[5] T. Kollo and D. von Rosen, Advanced Multivariate Statistics with Matrices. Springer, New York (2005).

[6] S. Kotz, N. Balakrishnan and N.L. Johnson, Continuous Multivariate Distributions. 2nd edition, Wiley, New York (2000) Vol. 1. 
[7] S. Kotz and S. Nadarajah, Multivariate $t$ Distributions and Their Applications. Cambridge University Press, Cambridge (2004).

[8] K.V. Mardia, Fisher's repeated normal integral function and shape distributions. J. Appl. Stat. 25 (1998) $231-235$.

[9] D.B. Owen, Handbook of Statistical Tables. Addison Wesley, Reading, Massachusetts (1962).

[10] B. Presnell and P. Rumcheva, The mean resultant length of the spherically projected normal distribution. Stat. Prob. Lett. 78 (2008) 557-563.

[11] H. Ruben, An asymptotic expansion for the multivariate normal distribution and Mills ratio. J. Res. Nat. Bureau Stand. B 68 (1964) 3-11.

[12] R. Savage, Mills ratio for multivariate normal distributions. Journal of Research of the National Bureau of Standards B 66 (1962) 93-96.

[13] G.P. Steck, Lower bounds for the multivariate normal Mills ratio. Ann. Prob. 7 (1979) 547-551.

[14] Y.L. Tong, The Multivariate Normal Distribution. Springer Verlag, New York (1990).

[15] D. von Rosen, Infuential observations in multivariate linear models. Scand. J. Stat. 22 (1995) 207-222.

[16] C.S. Withers, A chain rule for differentiation with applications to multivariate Hermite polynomials. Bull. Aust. Math. Soc. 30 (1984) 247-250.

[17] C.S. Withers, The moments of the multivariate normal. Bull. Aust. Math. Soc. 32 (1985) 103-108. 